\title{
Gamma rays from dark matter annihilation in the Draco and observability at ARGO
}

\author{
Xiao-Jun Bi, ${ }^{1, *}$ Hong-Bo Hu, ${ }^{1}$ and Xinmin Zhang $^{2}$ \\ ${ }^{1}$ Key laboratory of particle astrophysics, IHEP \\ ${ }^{2}$ Theoretical Physics Division, IHEP \\ Chinese Academy of Sciences, Beijing 100049, P. R. China
}

\begin{abstract}
The CACTUS experiment recently observed a gamma ray excess above $50 \mathrm{GeV}$ from the direction of the Draco dwarf spheroidal galaxy. Considering that Draco is dark matter dominated the gamma rays may be generated through dark matter annihilation in the Draco halo. In the framework of the minimal supersymmetric extension of the standard model we explore the parameter space to account for the gamma ray signals at CACTUS. We find that the neutralino mass is constrained to be approximately in the range between $100 \mathrm{GeV} \sim 400 \mathrm{GeV}$ and a sharp central cuspy of the dark halo profile in Draco is necessary to explain the CACTUS results. We then discuss further constraints on the supersymmetric parameter space by observations at the ground based ARGO detector. It is found that the parameter space can be strongly constrained by ARGO if no excess from Draco is observed above $100 \mathrm{GeV}$.
\end{abstract}

*Electronic address: bixj@mail.ihep.ac.cn 


\section{INTRODUCTION}

The existence of cosmological dark matter has been established by various astronomical observations. However, the evidences come mainly from the gravitational effects of the dark matter component. The nature of dark matter remains elusive and keeps one of the most outstanding puzzles in particle physics and cosmology [1]. The primordial nucleosynthesis and cosmic microwave background measurements constrain the baryon component and most dark matter component should be non-baryonic. The development in understanding the large scale structure formation requires the dark matter be cold. From the theoretical considerations the favored candidate for cold dark matter (CDM) seems to be the weakly interacting massive particles (WIMPs) [1] .

The WIMPs can be detected indirectly by observing the annihilation products, such as gamma rays, neutrinos, anti-protons and positrons. Exploring the anomalous results from the cosmic ray experiments is one viable way to identify the dark matter. Since the annihilation rate is proportional to the square of the dark matter density, the ideal sites for dark matter detection should have high dark matter density. The galactic center is believed to be a promising source of dark matter annihilation 2]. However, the existence of the central supermassive black hole and the supernova remnant Sgr A* contaminates the dark matter signals heavily . Alternative sites, such as the substructures of the Milky Way or the dark matter dominated dwarf spheroidal galaxies (dSph), have been studied in the $\operatorname{Refs}[3,44,5,6,6]$.

Recently, the CACTUS gamma-ray experiment reported an excess of gamma rays from the direction of Draco, a nearby dSph 8]. Since Draco is dark matter dominated and no other gamma ray sources are expected to be hosted [9, 10] the excess has been attributed to the annihilation of dark matter in the Draco halo [11, 12]. The results are still preliminary and, if confirmed, will have important implications on the nature of dark matter and the density profile of Draco. Additional observations of the signal by other experiments is therefore very important. The GLAST [13], a satellite-based experiment, and the MAGIC [14], a groundbased Atmospheric Čerenkov Telescope (ACT), have been considered to check the CACTUS results [11, 12]. In the present work, we will discuss a possibility of detecting or constraining the gamma rays observed by CACTUS at ARGO [15], a ground-based extensive air shower (EAS) detector. 
In the next section we will first give the general formula for dark matter annihilation. Then we will discuss the implications of CACTUS results on the gamma ray spectrum and fluxes in Sec III. The sensitivity of ARGO is given in Sec. IV and the numerical results are presented in Sec. V. We conclude in Sec. VI.

\section{GAMMA RAYS FROM DARK MATTER ANNIHILATION}

The annihilation of two WIMPs can produce the continuous spectrum of gamma rays arising mainly in the decays of the neutral pions produced in the fragmentation processes initiated by the tree level final states. The fragmentation and decay processes can be simulated with the Pythia package 16$]$.

The annihilation rate in unit time and unit volume is given by

$$
R=\langle\sigma v\rangle n^{2} / 2=\frac{\langle\sigma v\rangle \rho^{2}}{2 m^{2}}
$$

where $\sigma$ and $v$ are the annihilation cross section and the relative velocity of the two dark matter particles respectively, $n$ and $\rho$ are the number and mass densities of dark matter and $m$ is its mass, the factor 2 in the denominator arises due to the identical initial particles. We note that the annihilation rate is proportional to the square of the dark matter density and therefore, a high density region can greatly enhance the annihilation fluxes.

The gamma ray flux from the Draco halo is therefore given by

$$
\Phi_{\gamma}(E)=\phi^{\gamma}(E) \frac{\langle\sigma v\rangle}{2 m^{2}} \frac{\int d V \rho^{2}}{4 \pi D^{2}}=\frac{\phi^{\gamma}(E)}{4 \pi} \frac{\langle\sigma v\rangle}{2 m^{2}} \times \frac{1}{D^{2}} \int_{\Delta \Omega} d \Omega \int 4 \pi r^{2} d r \rho^{2}(r)
$$

where the halo profile is assumed approximately spherically symmetric with the density profile $\rho(r), D=75.8 \pm 0.7 \pm 5.4 \mathrm{kpc}$ is the distance to Draco [17], $\phi^{\gamma}(E)$ is the differential

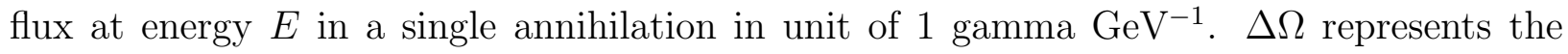
angular resolution of the detector.

The density profile $\rho(r)$ of Draco is constrained by observations. A recent analysis shows that both a cored and a cuspy profile, such as the NFW profile [18], are consistent with the observational data and the results of N-body simulation [19]. The 'astrophysical factor' in Eq. (21) defined as

$$
\Phi_{\text {astro }}=\frac{1}{D^{2}} \int_{\Delta \Omega} d \Omega \int 4 \pi r^{2} d r \rho^{2}(r)
$$


which is determined by the astrophysical quantities solely, is severely constrained by observational data. It is found that $\Phi_{\text {astro }}$ varies by a factor of approximately only 200 , i.e., $\Phi_{\text {astro }} \cong\left(3.2 \times 10^{-4} \sim 6.4 \times 10^{-2}\right) \mathrm{GeV}^{2} \mathrm{~cm}^{-6} \mathrm{kpc}$ sr following Ref. [19].

The other part in Eq. (21) is determined by particle physics which defines the nature of dark matter. We will calculate the 'particle factor' in the framework of the minimal supersymmetric standard model (MSSM). The MSSM is the most attractive model beyond the standard model of particle physics. In the R-parity conserved MSSM, the lightest supersymmetric particle, the lightest neutralino, provides a natural candidate for WIMP. The MSSM is well defined by a set of free parameters, which lead to the uncertainties in predicting the gamma ray flux from the particle physics. Once the particle factor is determined and combine with the astrophysical factor given above, we can give the predicted gamma ray flux from Draco.

\section{THE CACTUS EXPERIMENT}

CACTUS is a ground based Air Čherenkov Telescope (ACT) located at Solar Two near Barstow, California. CACTUS utilizes a set of 144 heliostats, each $42 \mathrm{~m}^{2}$, to form a composite mirror with a total effective area of about $6,000 \mathrm{~m}^{2}$. The threshold energy for gamma rays at CACTUS is about $50 \mathrm{GeV}$ and the effective area for $\gtrsim 200 \mathrm{GeV}$ gamma rays reaches about $50,000 \mathrm{~m}^{2}$.

Within the angular region of about $1^{\circ}$ centered around the direction of Draco, CACTUS has recently observed an excess of approximately 30,000 photons for 7 hours observation above the average background outside Draco[8]. The threshold energy of the photons is about $50 \mathrm{GeV}$. There is no significant excess observed if the cutoff energy is improved to about $150 \mathrm{GeV}$. Although the results are still preliminary, however, if confirmed, the implications on dark matter are significant. It is interesting to consider the implications of the CACTUS experimental results seriously due to our completely ignorance of the nature of dark matter. In this section we will study the implications for the gamma ray spectrum and flux from the CACTUS results.

The gamma events are given by

$$
N_{\gamma}^{\text {observed }}=\epsilon_{\Delta \Omega} \int_{E_{t h}, \Delta \Omega}^{m_{\chi}} A_{e f f}(E) \Phi(E) d E d \Omega d T
$$




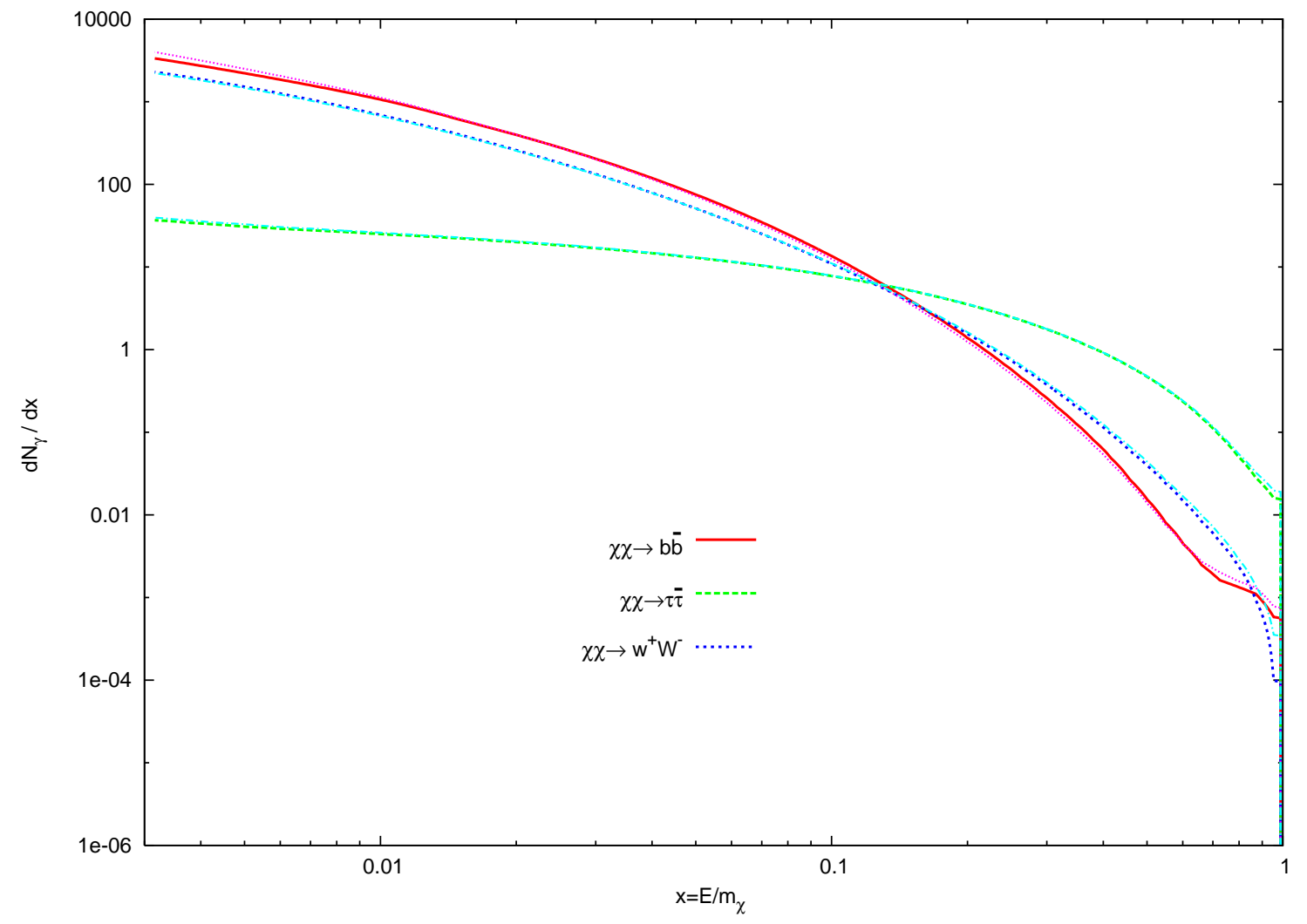

FIG. 1: The spectrum of gamma rays from neutralino annihilation, $\frac{d N_{\gamma}}{d x}$ with $x=E_{\gamma} / m_{\chi}$, for the final state of $W^{+} W^{-}, b \bar{b}$ and $\tau \bar{\tau} . m_{\chi}=100,500 \mathrm{GeV}$ has been taken which gives almost identical spectrum $\frac{d N_{\gamma}}{d x}$ for each final state.

where $\epsilon_{\Delta \Omega}=0.68$ is the fraction of signal events within the angular resolution of the instrument and the integration is for the energies above the threshold energy $E_{t h}$ and below the mass of neutralino, $m_{\chi}$, within the angular resolution of the instrument $\Delta \Omega$ and for the observational time. The effective area $A_{e f f}$ is a function of energy and $\Phi(E)=\phi_{0} \frac{d N_{\gamma}}{d E}$ is the flux of $\gamma$-rays from DM annihilation with $\phi_{0}$ the intensity normalization and $\frac{d N_{\gamma}}{d E}$ the shape of the spectrum. The effective area of CACTUS, which is energy dependent, can be parametrized as

$$
A_{\mathrm{eff}} \approx 47,000 \mathrm{~m}^{2}\left[1-\mathrm{e}^{-0.014\left(\mathrm{E}_{\gamma}-39.6 \mathrm{GeV}\right)}\right]+11.9 \times \mathrm{E}_{\gamma}(\mathrm{GeV})
$$

due to the simulation results [8].

From Eq. (4), we can see that in order to obtain the gamma ray flux from the observed event number we have to assume the gamma ray spectrum first. The spectrum of the gamma rays through neutralino annihilation depends on the final states into which the neutralinos 
have annihilated. In Fig. [ 1 we show the spectrum of gamma rays for the final states of gauge bosons $\chi \chi \rightarrow W^{+} W^{-}$and for the final states of $\chi \chi \rightarrow b \bar{b}$ and $\chi \chi \rightarrow \tau \bar{\tau}$, which represent the two extreme cases that the annihilated gamma rays have soft and hard spectra respectively. In the figure we have plotted the spectrum for $m_{\chi}=100,500 \mathrm{GeV}$ respectively. We find the spectrum, expressed as a function of a dimensionless quantity $x=E_{\gamma} / m_{\chi}$, is not sensitive to the mass of the neutralino, $m_{\chi}$.

The integrated gamma ray flux above the threshold energy of $50 \mathrm{GeV}$ is given by

$$
I_{\gamma}(>50 \mathrm{GeV})=\int_{50 \mathrm{GeV}}^{m_{\chi}} \Phi(E) d E=\int_{50 \mathrm{GeV}}^{m_{\chi}} \frac{d N_{\gamma}}{d E} d E \cdot \frac{N_{\gamma}^{\text {observed }}}{\epsilon_{\Delta \Omega} \int_{50 \mathrm{GeV}}^{m_{\chi}} A_{e f f}(E) \frac{d N \gamma}{d E} d E \cdot T},
$$

where we have assumed that the effective area has no zenith angle dependence within the angular resolution. From this equation we know the softer the spectrum the greater the gamma ray flux since $A_{e f f}$ is small at low energies. For soft spectrum, taking $m_{\chi}=100 \mathrm{GeV}$ and $b \bar{b}$ final states, we get $I_{\gamma}(>50 \mathrm{GeV})=1.7 \times 10^{-8} \mathrm{~cm}^{-2} \mathrm{~s}^{-1}$, while for the hard spectrum, taking $m_{\chi}=300 \mathrm{GeV}$ and $\tau \bar{\tau}$ final states, we get $I_{\gamma}(>50 \mathrm{GeV})=7.3 \times 10^{-9} \mathrm{~cm}^{-2} \mathrm{~s}^{-1}$. This spectrum is taken in order not to give too much excess above $150 \mathrm{GeV}$. Concerning the uncertainties from the noise rejection procedures, the misidentification of the electronic and hadronic primary events and that the angular region of CACTUS is larger than that of Draco, the observed excess may be greatly larger than the real signal of dark matter annihilation. Therefore in our theoretical calculation we make an assumption that the uncertainty of the gamma ray flux is larger than the current CACTUS data by relaxing the lower bound by an order of magnitude. We finally get the gamma ray flux from Draco which is approximately in the range of

$$
7.3 \times 10^{-10}<I_{\gamma}(>50 \mathrm{GeV})<1.7 \times 10^{-8} \mathrm{~cm}^{-2} \mathrm{~s}^{-1} .
$$

Since there is no significant excess observed above $150 \mathrm{GeV}$ the gamma ray spectrum is further constrained. We assume that the events above $150 \mathrm{GeV}$ do not exceed the Possion fluctuation of the background, which includes the misidentification of hadronic cosmic rays as gamma signals, the electronic comic ray events and the galactic diffuse gamma rays. We have adopted the expressions as

$$
\phi_{h}(E)=1.49 E^{-2.74} \mathrm{~cm}^{-2} \mathrm{~s}^{-1} s r^{-1} G e V^{-1}
$$

for the hadronic contribution [20],

$$
\phi_{e}(E)=6.9 \times 10^{-2} E^{-3.3} \mathrm{~cm}^{-2} \mathrm{~s}^{-1} s r^{-1} \mathrm{GeV}^{-1}
$$


for the electronic contribution [21],

$$
\phi_{\text {galac }-\gamma}(E)=8.56 \times 10^{-6} E^{-2.7} \mathrm{~cm}^{-2} s^{-1} s r^{-1} \mathrm{GeV}^{-1}
$$

for the Galactic $\gamma$-ray emission at the direction of Draco $\left(l=86.4^{\circ}, b=34.7^{\circ}\right)$, extrapolated from the EGRET data at low energies [22].

In principle the gamma ray flux above $150 \mathrm{GeV}$ also depends on the spectrum of the gamma ray. However, due to Eq. (5) the effective area above $150 \mathrm{GeV}$ is not so sensitive to energy differing from that at energies below $100 \mathrm{GeV}$. Considering the large systematic uncertainties and the possible problems in the noise reduction procedure we approximate the effective area above $150 \mathrm{GeV}$ as $50,000 \mathrm{~m}^{2}$, being a constant. Then we get a conservative upper limit of $I_{\gamma}(>150 \mathrm{GeV})$. Assuming that about 90 percent of the hadronic comic ray background can be rejected within the angular region due to the different shape of the Čherenkov wavefront induced by electronic and hadronic showers, we get that

$$
I_{\gamma}(>150 \mathrm{GeV}) \lesssim 3 . \times 10^{-11} \mathrm{~cm}^{-2} \mathrm{~s}^{-1}
$$

In the next sections we will explore the supersymmetric (SUSY) parameter space to account for the gamma excess observed at CACTUS taking into account the constraints given by the Eqs. (71) and (11).

\section{SENSITIVITY OF ARGO}

The ARGO-YBJ experiment, locates at YangBaJing (90.522 ${ }^{\circ}$ east, $30.102^{\circ}$ north, $4300 \mathrm{~m}$ a.s.l) in Tibet, China, is a ground-based telescope optimized for the detection of small size air showers. The energy threshold of the detector is designed to be about $100 \mathrm{GeV}$. The detector consists of a single layer of RPCs floored in a carpet structure covering an area of $\sim 10^{4} \mathrm{~m}^{2}$. The detector is under construction and the central carpet will be completed early in 2006 and put in stable data taking soon after.

The performances of the detector have been studied by means of Monte Carlo simulations 23]. Defined as a product of the sampling area and the trigger efficiency, the effective area characterizes the power of the detector in recording the number of events for a given energy and time interval from a given direction. For both primary $\gamma$ and hadrons with energy near the threshold, the effective area can be approximately parameterized as $A_{\text {eff }} \approx A_{100} E^{2.4}$, 
when the trigger condition is set to be larger than or equal to 20 fired pads, where $A_{100} \sim$ $100 \mathrm{~m}^{2}$ is the effective area for primary $\gamma$ ray events at the threshold energy of about 100 $\mathrm{GeV}$ [23]. Above the threshold energy the effective area increases rapidly and reaches about $10,000 \mathrm{~m}^{2}$ for $\mathrm{TeV}$ gamma rays. At the same time, simulation also shows that at low energies the protons have lower trigger efficiency than photons. The effective area for protons near the threshold energy is about one order of magnitude smaller than that of gamma, leading to a great suppression of the background.

The Draco dSph is within the field of view of the ARGO detector with the closest zenith angle to be $\sim 27^{\circ}$. Following up observations on the gamma excess seen by CACTUS have been considered at GLAST and MAGIC [11, 12]. Ground-based extensive air shower (EAS) arrays with low energy threshold, such as ARGO [15] and the next generation all-sky high energy gamma-ray telescope HAWC [24], have complementary properties to the satellite borne experiments and the ACTs. They have large effective areas and at the same time possess the advantages in large field of view and near $100 \%$ duty cycle. However, the EAS arrays usually have a poorer hadron-photon identification power. In this work, we will discuss how to constrain the gamma ray signal from Draco by the ARGO experiment.

For this purpose, we focus on the events for the energy below $\sim 400 \mathrm{GeV}$, since we will see in the next section that the CACTUS excess constrains the neutralino mass to be lower than $\sim 400 \mathrm{GeV}$. The number of background events for one year's data taking at ARGO is therefore also estimated in this energy range. To constrain the signal at the $2 \sigma$ level for one year's observation, the flux above $50 \mathrm{GeV}$ from Draco is then constrained as

$$
I_{\gamma}(>50 G e V)=\frac{2 \sqrt{N_{b k g}}}{A_{100} T} \cdot \frac{\int_{50}^{m_{\chi}} \frac{d N_{\gamma}}{d E} d E}{\epsilon_{\Delta \Omega} \int_{100}^{m_{\chi}}\left(\frac{E}{100}\right)^{2.4} \frac{d N_{\gamma}}{d E} d E},
$$

where again the zenith angle dependence of the effective area of the ARGO detector is ignored.

\section{NUMERICAL RESULTS}

In this section we will explore the SUSY parameter space to account for the CACTUS excess assuming that the excess (or a fraction of the excess) is generated by neutralino annihilation in the Draco halo. The constraint on the parameter space from ARGO is taken into account. 


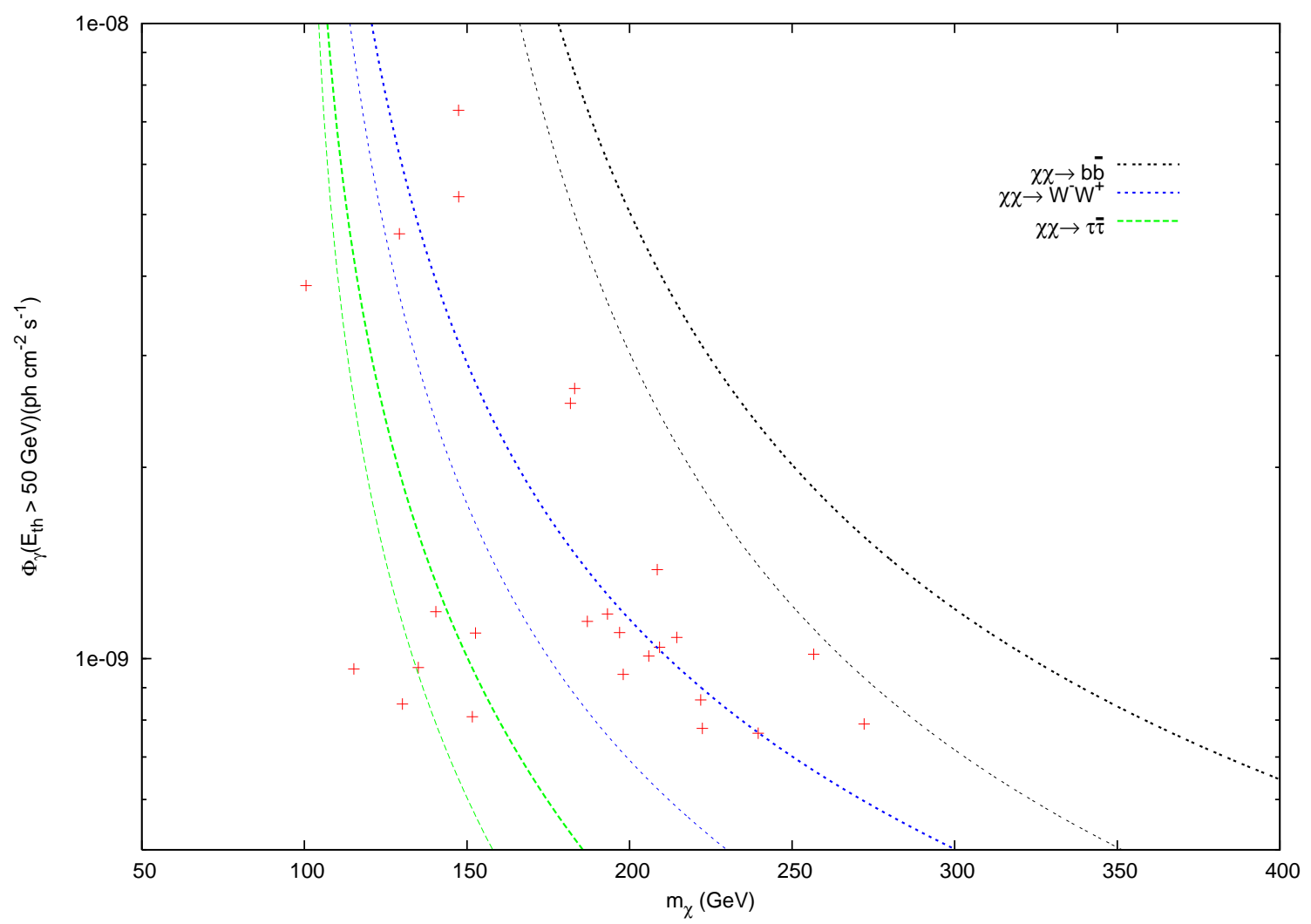

FIG. 2: The integrated $\gamma$-ray fluxes by neutralino annihilation from Draco above the threshold energy of $50 \mathrm{GeV}$ as a function of the neutralino mass. The fluxes are given within the angular resolution of $\Delta \Omega=10^{-3}$. Each point in the figure represents a set of low energy SUSY parameters which survive all the current limits. A boost factor 10 relative to the maximal astrophysical factor derived from [19] has been assumed. The lines shows the $2 \sigma$ constraints from the ARGO experiment assuming a $W^{+} W^{-}, b \bar{b}$ or $\tau \bar{\tau}$ final state with or without gamma/hadron discrimination.

The R-parity conserved MSSM is described by more than one hundred parameters describing the soft supersymmetry breaking. However, for the processes related with dark matter production and annihilation, only several parameters are relevant under some simplifying assumptions, i.e., the higgsino mass parameter $\mu$, the bino mass parameter $M_{1}$, the wino mass parameter $M_{2}$, the mass of the CP-odd Higgs boson $m_{A}$, the ratio of the Higgs vacuum expectation values $\tan \beta$, the scalar fermion mass parameter $m_{\tilde{f}}$, the trilinear soft breaking parameter $A_{t}$ and $A_{b}$. To determine the low energy spectrum of the SUSY particles and coupling constants, the following assumptions have been made: all the sfermions have common soft-breaking mass parameters $m_{\tilde{f}}$; all trilinear parameters are zero except those of 


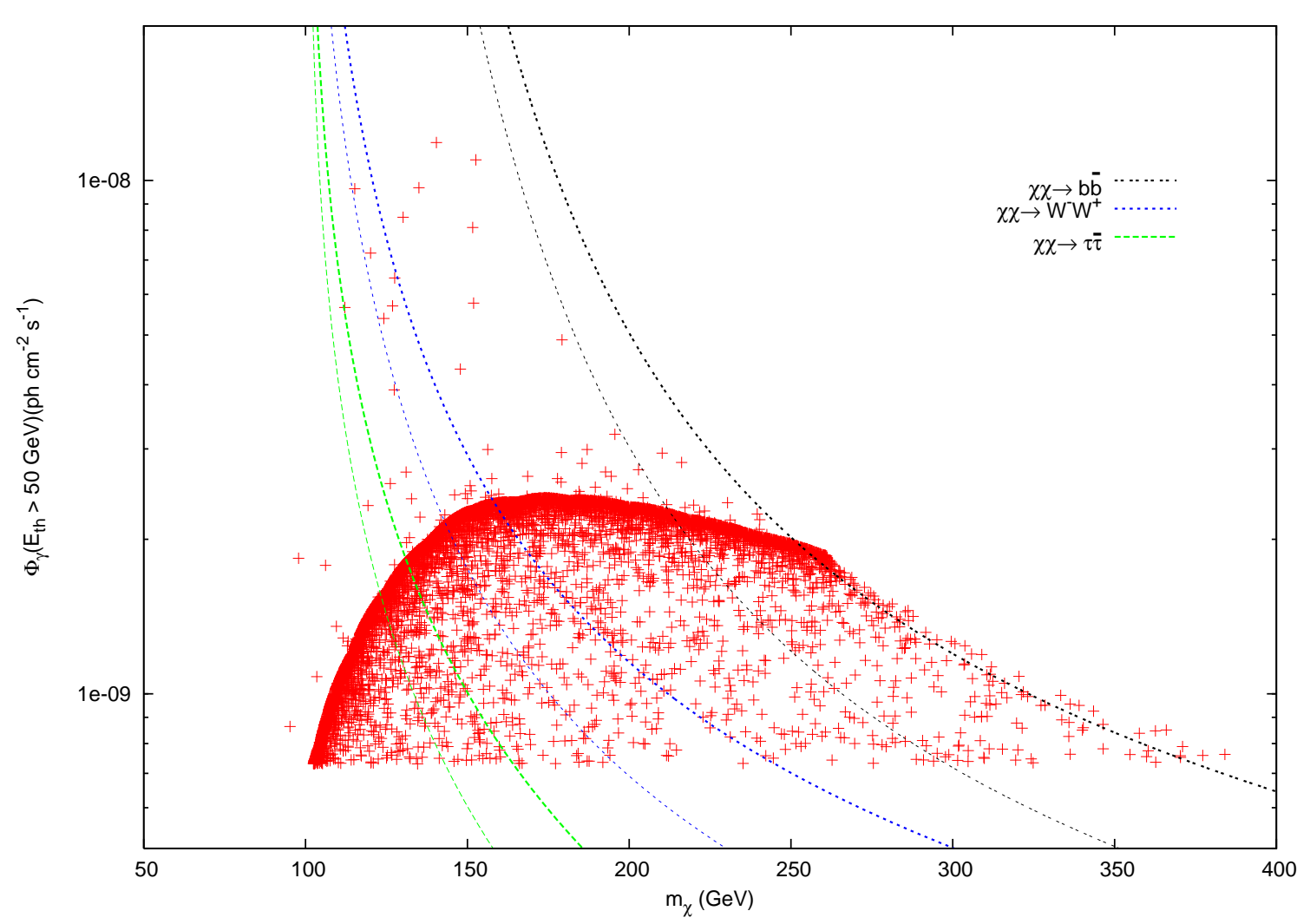

FIG. 3: Same as Fig. 2 except that a boost factor of 100 has been assumed.

the third family; the gluino and wino have the mass relation, $M_{3}=\left(\alpha_{s}\left(M_{Z}\right) / \alpha_{e m}\right) \sin ^{2} \theta_{W} M_{2}$, coming from the unification of the gaugino mass at the grand unification scale. However, to explore more general low energy phenomenological SUSY parameter space we relax the relationship between $M_{1}$ and $M_{2}$ derived from the grand unification scale.

We perform a numerical random scanning in the 8-dimensional supersymmetric parameter space using the package DarkSUSY [26]. The ranges of the parameters are as following: $50 \mathrm{GeV}<|\mu|, M_{2}, M_{A}, m_{\tilde{f}}<5 T e V, 1.1<\tan \beta<60,-3 m_{\tilde{q}}<A_{t}, A_{b}<3 m_{\tilde{q}}$, $\operatorname{sign}(\mu)= \pm 1$. The parameter space is constrained by the theoretical consistency requirements, such as the correct symmetry breaking pattern, the neutralino being the LSP and so on. The accelerator data constrains the parameter further from the spectrum requirement, the invisible Z-boson width, the branching ratio of $b \rightarrow s \gamma$ adopting the experimental data given by the Particle Data Group in the year of 2002 [27].

Another important constraint comes from cosmology. Combining the recent observation data on cosmic microwave background, large scale structure, supernova and data from HST Key Project the cosmological parameters are determined quite precisely. Especially, the 


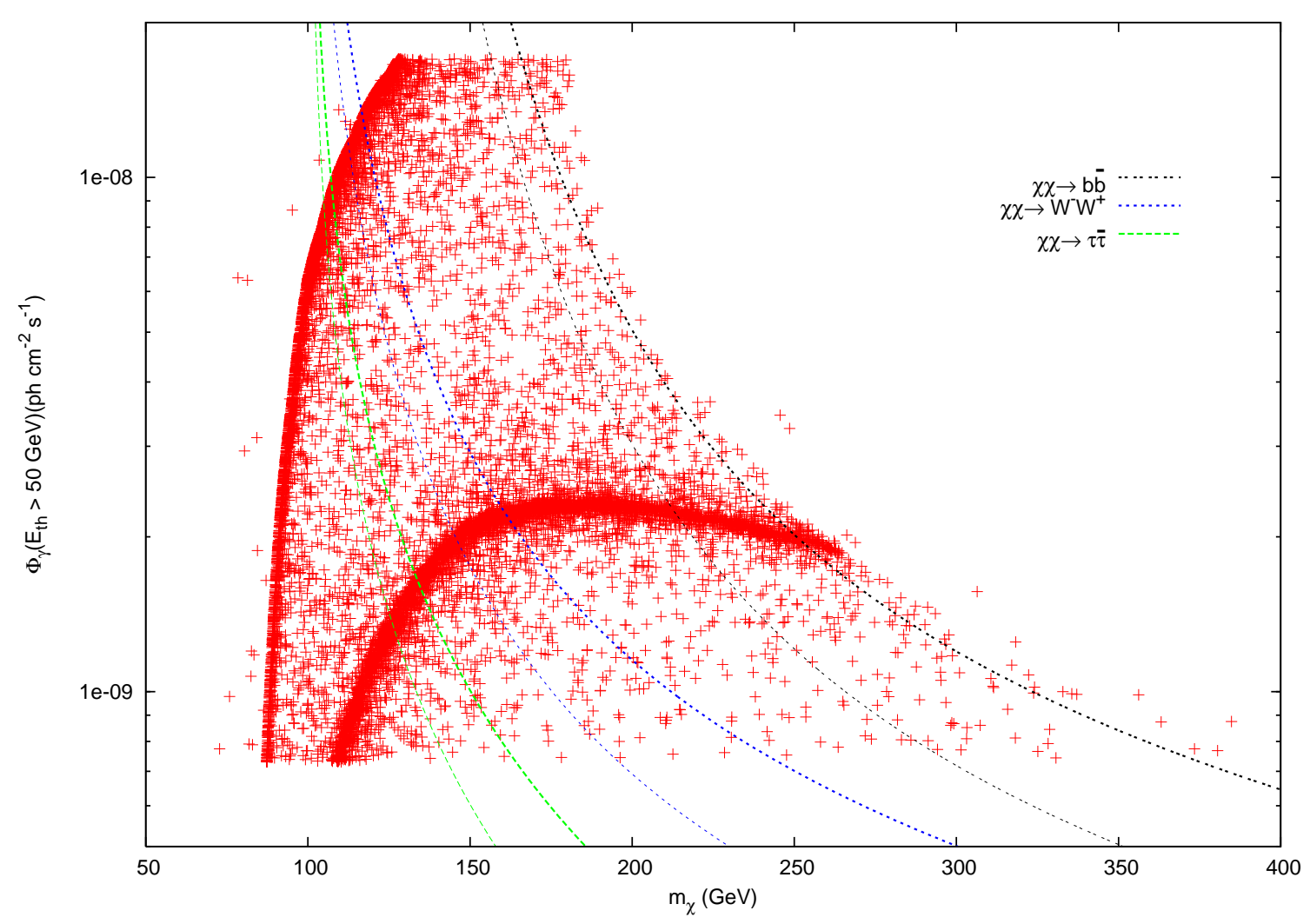

FIG. 4: Same as Fig. 2 except that a boost factor of 1000 has been assumed.

abundance of the cold dark matter is given by [28] $\Omega_{\mathrm{CDM}} h^{2}=0.113_{-0.009}^{+0.008}$. We constrain the SUSY parameter space by requiring the relic abundance of neutralino $0<\Omega_{\chi} h^{2}<$ 0.137, where the upper limit corresponds to the $3 \sigma$ upper bound from the cosmological observations. When the relic abundance of neutralino is smaller than a minimal value the thermally produced neutralino represents a subdominant dark matter component. We assume non-thermal mechanism to give the correct dark matter relic density [29]. The effect of coannihilation between the fermions is taken into account when calculating the relic density numerically.

We find that a 'boost factor' at the order of $10 \sim 1000$ is necessary to account for the CACTUS results. The 'boost factor' means that the astrophysical factor calculated by a cored or a cuspy profile in the Sec II should be enhanced by this factor to give the observed flux. The 'boost factor' requires a much sharper density profile compared with the NFW profile, such as a Moore profile [30] or a spike profile due to the existence of an intermediate mass central black hole [31] in Draco.

In Figs. 2, 3] and 4, we plot the integrated $\gamma$-ray fluxes above the threshold energy $50 \mathrm{GeV}$ 
within the solid angle $\Delta \Omega=10^{-3}$ as a function of the neutralino mass. The results in Figs. 2. 3] and 4 have enhanced the astrophysical factor by a boost factor of 10, 100 and 1000 respectively. Each point in the figure corresponds to a model with a set of definite SUSY parameters in the 8-dimensional parameter space which can explain the CACTUS results constrained by Eqs. (71) and (11) and allowed by all other collider and cosmology constraints. The scatter of the points represents the uncertainty coming from the unknown soft SUSY breaking parameters. The lines represent the $2 \sigma$ constraints of ARGO by assuming the final states being the $W^{+} W^{-}, b \bar{b}$ or $\tau \bar{\tau}$. For the upper set of line we have assumed no hadron/photon discrimination at all, while for the lower set of lines we assume a part of hadrons are rejected based on neural network so that the significance of detection is improved by a quality factor of 1.6 [32]. From the figure we can see that if no excess is observed at ARGO above $100 \mathrm{GeV}$ a large part of the parameter space is constrained.

It is worthwhile commenting on the results here. First, if extending the gamma spectrum to lower energies, we find the CACTUS result is difficult to reconcile with the EGRET result 33] which did not observe excess at the direction of Draco between $1 \sim 10 \mathrm{GeV}$. Therefore a hard spectrum is expected to reconcile the EGRET and the CACTUS results, which requires the dominant annihilation product be $\tau \bar{\tau}$ [11]. The hard spectrum leads to more opportunities to observe the signal in ARGO which can be seen from the Figs. 2, 3] and 4. Alternatively one would assume that only about 1 percent of the present excess is real signal from the annihilation of the dark matter. In this case we find parameter space to account for the signal and be consistent with EGRET result in the range of $250 \mathrm{GeV}<m_{\chi}<800 \mathrm{GeV}$. The parameter space can be constrained by ARGO only for the $\tau \bar{\tau}$ final states. Second, the CACTUS result may also imply a monochromatic gamma spectrum at the energy of about $50 \mathrm{GeV}$. However, it is found that the branching ratio that two neutralino annihilate into two photons should be more than a half to be consistent with the EGRET result, which is incompatible with the SUSY model [12]. Finally, if we assume that only about $\lesssim 1 \%$ of the excess comes from DM annihilation, the signal can be explained without introduce any 'boost factor' if taking the nonthermal mechanism into account. This may be a natural assumption, while the confirmation of the gamma events from DM annihilation requires an instrument with better angular resolution, such as GLAST [12] to suppress the background. 


\section{SUMMARY AND CONCLUSION}

In this paper we have discussed the possibility of constraining the signal observed by the CACTUS experiment at the ground based EAS detector, ARGO. We assume the excess of gamma rays observed at CACTUS is produced by supersymmetric dark matter annihilation. We then explore the SUSY parameter space to give signal consistent with the CACTUS result and discuss the possibility to constrain the parameter space at ARGO. Our calculation shows that, depending on the gamma spectrum, ARGO will be able to constrain a large part of the parameter space if no signal is detected for one year observation.

If the CACTUS signal is finally confirmed, the implication on dark matter is dramatic. The central cusp of the dark halo at Draco should be much sharper than that of a NFW profile. The neutralino mass should be at the range of $100 \sim 400 \mathrm{GeV}$ to explain the signal of CACTUS. Furthermore, the spectrum of the annihilation gamma ray should be very hard in order to be consistent with the EGRET null result at the direction of Draco at the energy range between $1 \mathrm{GeV}$ and $10 \mathrm{GeV}$.

\section{Acknowledgments}

We thank Xuelei Chen for helpful discussions. This work is supported in part by the NSF of China under the grant Nos. 10575111, 10105004, 10120130794, 90303004.

[1] G. Jungman, M. Kamionkowski and K. Griest, Phys. Rept. 267 (1996) 195; G. Bertone, D. Hooper and J. Silk, Phys. Rept. 405, 279 (2005).

[2] L. Bergström, P. Ullio and J. H. Buckley, Astropart. Phys. 9, 137 (1998).

[3] E. A. Baltz, C. Briot, P. Salati, R. Taillet, J. Silk, Phys. Rev. D 61, 023514 (2000), arXiv:astro-ph/9909112.

[4] N. Fornengo, L. Pieri and S. Scopel, Phys. Rev. D 70, 103529 (2004), arXiv:hep-ph/0407342.

[5] N. W. Evans, F. Ferrer and S. Sarkar, Phys. Rev. D 69, 123501 (2004), arXiv:astro-ph/0311145.

[6] X.J. Bi, arXiv:astro-ph/0510714

[7] S.M. Koushiappas, A.R. Zentner, T. P. Walker, Phys. Rev. D 69, 043501 (2004). 
[8] P. Marleau, TAUP, Zaragoza, Spain, September 2005; M. Tripathi, Cosmic Rays to Colliders 2005, Prague, Czech Republic, September 2005; TeV Particle Astrophysics Workshop, Batavia, USA, July 2005; M. Chertok, proceedings of PANIC 05, Santa Fe, USA, October 2005.

[9] L.M. Young, Astron. J. 117, 1758 (1999).

[10] C. Tyler, Phys. Rev. D 66 (2002) 023509, arXiv:astro-ph/0203242.

[11] L. Bergström, D. Hooper, arXiv:hep-ph/0512317.

[12] S. Profumo, M. Kamionkowski, arXiv:astro-ph/0601249.

[13] A.Morselli et al., Proc. of the 32nd Rencontres de Moriond (1997).

[14] C. Baixeras et al., Nucl. Phys. Proc. Suppl., 114, 247 (2003).

[15] A. Aloisio et al., Nuovo Cim. 24C, 739 (2001); G. Di Sciascio et al., [ARGO-YBJ Collaboration], AIP Conf. Proc. 745, 663 (2005).

[16] T. Sjöstrand et al., Comput. Phys. Commun. 135, 238 (2001).

[17] A. Z. Bonanos, K. Z. Stanek, A.H. Szentgyorgyi, D.D. Sasselov and G.A. Bakos, Astrophys. J. 127, 821 (2004).

[18] J. F. Navarro, C. S. Frenk, and S. D. M. White, Mon. Not. R. Astron. Soc. 275, 56 (1995); J. F. Navarro, C. S. Frenk, and S. D. M. White, Astrophys. J. 462, 563 (1996); J. F. Navarro, C. S. Frenk, and S. D. M. White, Astrophys. J. 490, 493 (1997).

[19] S. Mashchenko, H. M. P. Couchman, A. Sills, Astrophys. J. 624, 726 (2005). S. Mashchenko, A. Sills and H.M.P. Couchman, arXiv:astro-ph/0511567

[20] T. Gaisser et al., Proc. of the 27th ICRC (2001).

[21] M.S. Longair, High Energy Astrophysics, Cambridge Universeity Press (1992).

[22] L. Bergström, P. Ullio, J. Buckley, Astropart. Phys. 9, 137 (1998).

[23] S.W. Cui, H.B. Hu, Proc. of the 29th ICRC (2005); X.X. Zhou et al. Proc. of the 29th ICRC (2005).

[24] G. Sinnis, A. Smith, J.E. McEnery, astro-ph/0403096.

[25] N. Fornengo, L. Pieri, S. Scopel, Phys. Rev. D 70, 103529 (2004).

[26] P. Gondolo, J. Edsjo, P. Ullio, L. Bergstrom, M. Schelke, E.A. Baltz, JCAP 0407, 008 (2004), astro-ph/0406204

[27] K. Hagiwara et al., Phys. Rev. D 66, 010001 (2002).

[28] C. L. Bennett et al., Astrophys. J. Suppl. 148, 1 (2003), arXiv: astro-ph/0302207, D. N. Spergel et al., Astrophys. J. Suppl. 148, 175 (2003), arXiv: astro-ph/0302209. 
[29] R. Jeannerot, X. Zhang and R. H. Brandenberger, JHEP 9912, 003 (1999); T. Moroi and L. Randall, Nucl. Phys. B 570, 455 (2000); W. B. Lin, D. H. Huang, X. Zhang and R. H. Brandenberger, Phys. Rev. Lett. 86, 954 (2001); B. Murakami and J. D. Wells, Phys. Rev. D 64, 015001 (2001); M. Fujii and K. Hamaguchi, Phys. Lett. B 525, 143 (2002); G. B. Gelmini, P. Gondolo, arXiv: hep-ph/0602230.

[30] B. Moore, S. Ghigna, F. Governato, G. Lake, T. Quinn, J. Stadel, P. Tozzi, 1999, ApJ, 524, L19.

[31] P. Gondolo, J. Silk, Phys. Rev. Lett. 83, 1719 (1999); G. Bertone, A. R. Zentner and J. Silk, Phys. Rev. D 72, 103517 (2005), arXiv:astro-ph/0509565

[32] The ARGO-YBJ Project (addendum to the Proposal), ARGO-YBJ collaboration.

[33] http://cossc.gsfc.nasa.gov/egret/ 